\title{
EFECTO DEL ESTADO DE MADUREZ Y LA FERTILIZACIÓN NITROGENADA SOBRE LA PRODUCCIÓN Y VALOR NUTRITIVO DEL PASTO TRANSVALA (Digitaria decumbens cv. Transvala) PARA HENIFICACIÓN BAJO CONDICIONES DE SECANO.
}

\author{
Jorge L. Morales , Argerie Cruz , Vidal Acuña ${ }^{1}$
}

\begin{abstract}
RESUMEN
La producción y la calidad del pasto transvala (Digitaria decumbens cv. Transvala) para heno, dependen de la edad de rebrote y la fertilización. El presente estudio tuvo como objetivo determinar la producción y calidad del pasto cuando se cosecha a los 40, 50 y 60 días de rebrote a tasas de fertilización nitrogenada (nitrato de amonio) de 0, 105, 205, 305, 405 y $505 \mathrm{~kg}$ de N/ha/cosecha, en tres dosis, bajo condiciones de secano en el Pacífico Seco de Costa Rica. El estudio se ejecutó bajo un diseño de parcelas divididas con tres repeticiones. La evaluación se realizó del 11 de octubre al 12 de diciembre de 1999. La producción de forraje en kilogramos de materia seca por hectárea (kg MS/ha) aumenta $(3.741,4 ; 4.236,2$ y $4.839,8)$ con la edad de rebrote $(P<0,10)$ y alcanza un máximo $(4.847,5)$ cuando se fertiliza a una tasa de $105 \mathrm{~kg} \mathrm{~N} / \mathrm{ha}(\mathrm{P}<0,01)$. Se encontró una interacción entre edad de rebrote y tasas de fertilización $(P<0,05)$ para porcentaje de proteína cruda $(\% \mathrm{PC})$, en donde el aumento como efecto de la fertilización nitrogenada es menor conforme el forraje madura (días de rebrote) $40>50>60$, siendo de 8,$57 ; 7,90 ;$ y $7,63 \%$ PC respectivamente, a una tasa de fertilización de $105 \mathrm{~kg}$ de N/ha. Se concluye que bajo las características edafo-climáticas del área donde se encuentra ubicada la Estación Experimental Enrique Jiménez Núñez en Cañas, Guanacaste, en condiciones de secano, la fertilización nitrogenada del pasto transvala en forma de nitrato de amonio a tasas de $105 \mathrm{~kg} \mathrm{~N} / \mathrm{ha} / \mathrm{corte}$, permite aumentar la producción en más de 1,2 $t$ de forraje en base seca y el contenido de proteína cruda hasta 8,57\%. Por edad de rebrote, en términos de PC y producción, el rango más adecuado está entre 40 y 50 días.
\end{abstract}

Palabras clave: Forrajes, henificación, producción, calidad, fertilización.

\section{INTRODUCCIÓN}

La tecnología de la henificación es conocida desde hace muchos años en el mundo y Costa Rica no es la excepción. Sin embargo, ésta no es de amplio uso en el país porque está dirigida a paliar, principalmente, la baja disponibilidad de forraje durante el período seco; en épocas de temporal en el trópico húmedo y para el cuido de caballos pero no para la producción de leche o carne.

La producción de heno, de más de 400 mil pacas anuales, proviene principalmente de la región Chorotega de Costa Rica y data de hace más de 40 años. Aunque se utilizan varias especies forrajeras para henificar, el pasto transvala (Digitaria decumbens cv. Transvala (Boyd et al.1975) es la principal especie utilizada (Morales et al. 2003). La calidad de un forraje está determinada por su edad de rebrote y en general, las gramíneas forrajeras como el pasto transvala, mantienen esta característica aceptable (contenido de proteína, fibra, energía y digestibilidad) hasta los 45 días de edad de rebrote, a partir de la cual se reduce rápidamente hasta valores incompatibles para la producción animal.

'Instituto Nacional de Innovación y Transferencia en Tecnología Agropecuaria (INTA), Costa Rica. 
El sistema de producción de secano (crecimiento vegetativo durante invierno y cosecha en las primeras semanas de inicio del verano), proceso tradicional de producir heno en Costa Rica, permite altas producciones de pacas por la acumulación de forraje durante un período largo de hasta siete meses, pero por la misma razón de esa alta edad del forraje, su calidad es muy baja.

Se ha comprobado, que muchas veces el valor nutritivo de la paja de arroz es superior a la paca de transvala (Morales et al. 2003). Productores y comerciantes de estas pajas saben que sirven sólo para que los animales pierdan menos peso durante la época crítica y que se debe suministrar a los animales, además, melaza, urea, entre otros, de lo contrario no servirían ni para eso. Ni pensar que estas pajas sirvan para producir, tal vez como fuente de fibra en sistemas estabulados, particularmente aquellos de producción de leche. Tal parece que el poco desarrollo de esta actividad en el país se debe a problemas de calidad y que por ello exista una demanda real por estos forrajes sólo durante períodos críticos.

Dado que la calidad de los forrajes está directamente relacionado con su edad de rebrote y que en sistemas de secano de producción sólo se obtiene con certeza el corte de diciembre cuando llega el verano, la hipótesis que sustenta este trabajo de investigación es la factibilidad de mejorar significativamente la calidad y la producción del heno de pasto transvala, si se utilizan sistemas bajo riego.

Revertir las características de la henificación en Costa Rica hacia una industrialización del heno de calidad, permitiría al igual que en otros países, desarrollar una amplia actividad industrial y comercial con una gran contribución al desarrollo y eficiencia de segmentos de la ganadería bovina como la de engorde y lechería especializada, en sistemas de alimentación estabulada (EPA 2003; Leng 1991; Leng 1993). El presente es un estudio básico de respuesta del forraje a la fertilización nitrogenada y edad de rebrote, por lo que se realizó durante el invierno para adelantar información que servirá para realizar el estudio, propiamente de verano, con riego.

Por lo anterior, el objetivo de dicho estudio fue determinar la curva de producción y el valor nutritivo del pasto transvala, como respuesta a la fertilización nitrogenada y a la edad de rebrote.

\section{MATERIALES Y MÉTODOS}

\section{Ubicación del Ensayo}

El presente estudio se llevó a cabo en la Estación Experimental Enrique Jiménez Núñez del INTA, ubicada en el cantón de Cañas, en Guanacaste, $10 \mathrm{~km}$ al sureste del distrito central y $10 \mathrm{~km}$ al sur de la Carretera Panamericana y se encuentra a una altura de 9 -11 msnm.

Las condiciones edafo-climáticas de la localidad, se caracterizan por presentar suelos Mollisoles (Fluventic Haplusoll), profundos y fértiles, de textura franca aunque mal estructurados. Sus limitantes están relacionadas a la Zona de Vida de Bosque Húmedo, transición a Basal y Tropical, dadas por fuertes vientos y un patrón climático bi-modal, con un período seco de diciembre a abril y un invierno intenso en lluvias, de setiembre a mediados de noviembre, separados por un período que se ha manifestado errático en los últimos años, ligero de lluvias de mayo a mediados de julio y de aquí a agosto en el que se reduce la precipitación significativamente, correspondiente al veranillo de San Juan y a la canícula. Los suelos se caracterizan por tener bajos contenidos de fósforo, manganeso y potasio, aunque con una suma de cationes aceptable y $\mathrm{pH}$ de alrededor del 6,5 (Arroyo et al. 2005).

La Figura 1 muestra el comportamiento del clima en la zona (Hancock y Hargreaves 
1977), la cual está basada en un período de seis años y con una probabilidad de un $75 \%$ de que este patrón de lluvias pueda ocurrir.

La temperatura promedio de la zona ha sido de 24,6 $\mathrm{C}^{\circ}$; la humedad relativa $91 \%$; la precipitación máxima de $2.818 \mathrm{~mm}$; la precipitación mínima $1.018 \mathrm{~mm}$, durante el mismo período.

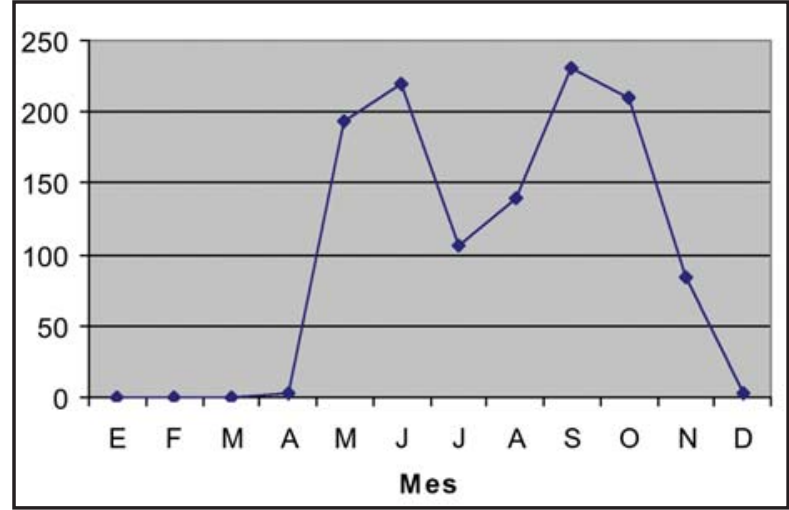

Figura 1. Patrón de lluvias con $75 \%$ probabilidades para el área de la Estación Experimental Enrique Jiménez Núñez (Hancock y Hargreaves 1977)

\section{Diseño y manejo experimental}

Las parcelas de evaluación del presente estudio, se establecieron en un campo de 3 ha de pasto transvala, sembrado en junio de 1999, el cual se adecuó para aplicar el riego por gravedad con el propósito de generar los estudios básicos necesarios, para el desarrollo de la henificación de alta calidad en Costa Rica.

El tamaño de la unidad experimental utilizada fue de $6 \mathrm{~m}^{2}$ (3 $\left.\mathrm{m} \times 2 \mathrm{~m}\right)$. Las parcelas fueron marcadas previo corte de uniformidad, a nivel del suelo. Posterior a la marcación de las parcelas, éstas recibieron $21 \mathrm{~kg} \mathrm{P} / \mathrm{ha}$ y $7 \mathrm{~kg} \mathrm{~N} / \mathrm{ha}$, en forma de fórmula completa (10-30-10) y un tercio de las dosis experimentales de nitrógeno. Las otras dos dosis de nitrógeno, se aplicaron a las diferentes parcelas a los 15 y 30 días posteriores de la primera aplicación, respectivamente.
EL estudio se arregló en un diseño estadístico de parcelas divididas, con bloques completos al azar y tres repeticiones, en un factorial con cinco niveles de fertilización nitrogenada y tres edades de rebrote. El análisis de los datos se hizo mediante el paquete estadístico SAS, utilizando la edad de rebrote, como parcela principal y los niveles de fertilización como sub-parcela.

Las dos variables independientes fueron: tasa de fertilización utilizada de 0, 105, 205, 305, 405 y $505 \mathrm{~kg} \mathrm{~N} / \mathrm{ha}$ y la edad de rebrote de 40, 50 y 60 días. Las variables dependientes fueron cuatro: producción de forraje en base seca, porcentaje de, materia seca (MS), proteína cruda (PC) y fibra en el forraje.

\section{Distribución al azar de las edades de rebrote y niveles de fertilización}

Los tres bloques ó repeticiones se ubicaron en tres sitios diferentes pero representativos de todo el campo de 3 ha de pasto transvala disponibles para los estudios. En cada bloque se asignaron los tres días de rebrote al azar y dentro de cada una de éstos se asignaron los niveles de fertilización, también al azar. Las parcelas se separaron a lo largo y ancho entre sí, con callejones de $2 \mathrm{~m}$ de ancho. Los niveles de fertilizante en cada parcela, correspondientes a los niveles por ha planeados, se dividieron en tres aplicaciones iguales, la primera el día cero y las siguientes a los 15 y 30 días posteriores. A estos tratamientos se les agregó una fórmula completa y se estableció un testigo absoluto (0A), al cual no se le aplicó ningún fertilizante.

Se debe considerar que estos resultados corresponden a la biomasa antes del proceso de henificación, durante el cual pueden ocurrir algunas pérdidas no contabilizables en el presente estudio. 


\section{Muestreos y análisis de laboratorio}

Se tomaron muestras a los 40, 50 y 60 días de rebrote cortando cada parcela a nivel del suelo. Se pesó toda la biomasa verde de la parcela con romana de reloj de $50 \mathrm{~kg}$. Posteriormente se tomó una sub-muestra de aproximadamente $500 \mathrm{gr}$ de material fresco, en bolsas de papel debidamente identificadas. Éstas fueron pesadas en romana granataria y se almacenaron en hieleras mientras se llevaron a laboratorio al cabo de dos días, en donde se sometieron a secado en horno a $60{ }^{\circ} \mathrm{C}$, durante 72 horas.

En el laboratorio de Piensos y Forrajes del INTA, las muestras se secaron en horno a $105^{\circ} \mathrm{C}$ durante 24 horas, para determinar materia seca y posteriormente se molieron en molino de martillos utilizando malla de 2 $\mathrm{mm}$. Las muestras se analizaron para proteína cruda $(\mathrm{PC})$, utilizando el método Kjeldahl, fibra ácido detergente (FAD) y fibra neutro detergente (FND), utilizando la metodología de Van Soest (Van Soest 1967).

\section{RESULTADOS Y DISCUSIÓN}

\section{Contenido de materia seca}

El contenido de materia seca del forraje, aumenta con la edad de rebrote de la planta, pasando de $21 \%$ a 40 días de edad, a prácticamente $29 \%$ a 60 días de edad (Cuadro 1).
Este resultado concuerda con los reportes de la literatura (Morales 1989; Van Soest 1983) y corresponde a una observación prácticamente universal, indicando que a mayor edad de rebrote mayor el contenido de materia seca, ó dicho de otra manera, menor el contenido de humedad del forraje.

El contenido de materia seca del forraje, también se vio afectado por la fertilización nitrogenada. En este caso, sin embargo, fue un efecto inverso, en donde ese contenido disminuyó con cada incremento en nitrógeno aplicado. Es decir, a mayor cantidad de nitrógeno aplicado mayor el contenido de humedad del forraje (Figura 2).

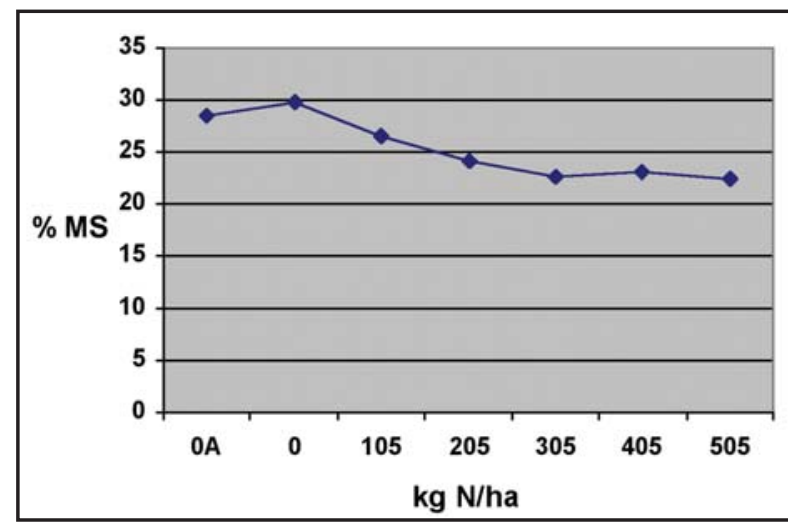

Figura 2. Efecto de diferentes niveles de fertilización sobre el contenido de materia seca del forraje. Estación Experimental Enrique Jiménez Núñez, Costa Rica. 1999. Estación Experimental Enrique Jiménez Núñez . Costa Rica. 1999.

Cuadro 1. Contenido de materia seca del forraje según edad de rebrote. Estación Experimental Enrique Jiménez Núñez, Costa Rica. 1999.

\begin{tabular}{ccccc}
\hline Variable & $\begin{array}{c}\text { Error estándar } \\
\text { de la media }\end{array}$ & & EDAD DE REBROTE (días) & \\
$\% \mathrm{MS}$ & 1,65 & 40 & 50 & 60 \\
\hline
\end{tabular}

$\% \mathrm{MS}=$ contenido de materia seca. Medias con letras diferentes son diferentes entre si $(P<0,01)$. 
Obsérvese que la fórmula completa aplicada, como única fuente de fertilizante, en el testigo cero tendió a aumentar el \% de materia seca en el forraje. Por el resultado pareciera que la aplicación de $\mathrm{P}$ y $\mathrm{K}$, acompañados con bajos niveles de $\mathrm{N}$, favorecen el aumento en el contenido de MS del forraje.

\section{Contenido de proteína cruda}

El comportamiento antagónico del efecto de la edad de rebrote (relación indirecta) y de la fertilización nitrogenada (relación directa), sobre el contenido de proteína cruda del forraje, resultó en una interacción significativa $(p<0,05)$ entre ambos, como se puede observar en la Figura 3.

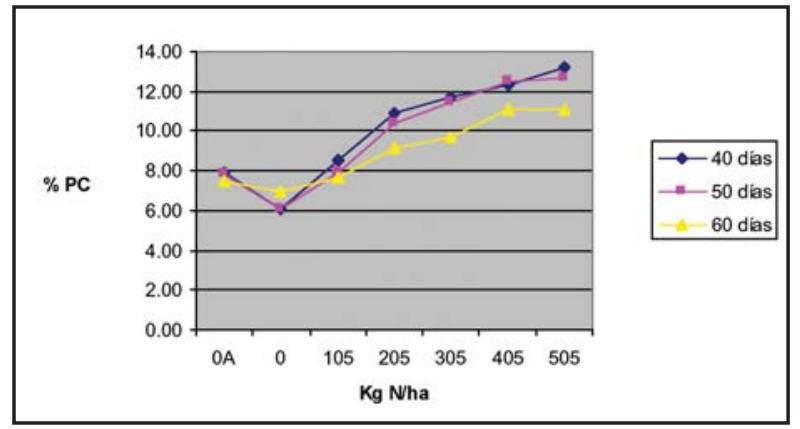

Figura 3. Contenido de proteína cruda del forraje como respuesta a la interacción de la edad del forraje con los niveles de fertilización. Estación Experimental Enrique Jiménez Núñez . Costa Rica. 1999.

Ésto quiere decir que, entre mayor sea la edad de rebrote del pasto, menor será el efecto positivo que tiene la fertilización nitrogenada sobre el contenido de proteína cruda del forraje (Milford y Minson 1966; NRC 1984). Dicho efecto fue particularmente evidente a edades de rebrote mayores a 50 días.

Es interesante rescatar que la fórmula completa a la tasa aplicada, provocó en el testigo cero en ausencia de suficiente nitrógeno, un efecto depresivo de la proteína en el forraje. Ésto en relación con el contenido de proteína del forraje del testigo absoluto.

\section{Producción de biomasa}

La producción de forraje en base seca resultó directamente relacionada con la edad de rebrote $(p<0,01)$, lo cual confirma observaciones similares obtenidas por (Leng 1993; Morales 1989) (ver Figura 4).

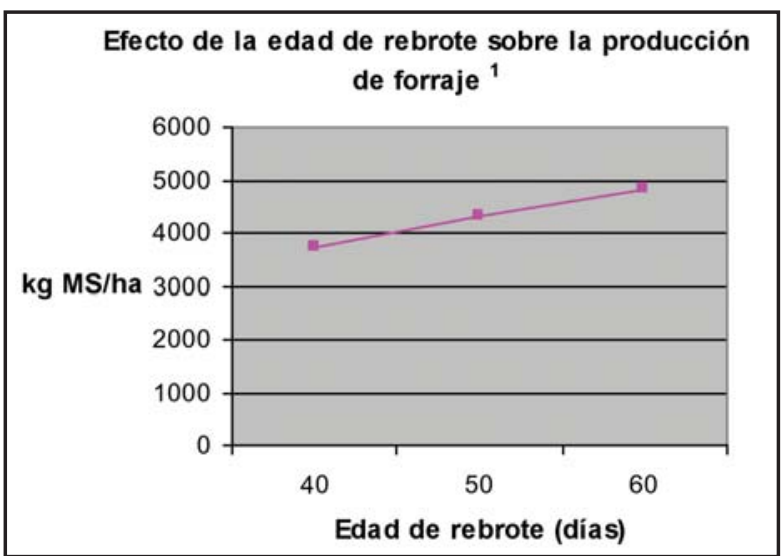

Figura 4. Efecto de la edad de rebrote sobre la producción de forraje. Estación Experimental Enrique Jiménez Núñez . Costa Rica. 1999.

En el presente estudio, en promedio, por cada aumento de 10 días en la edad de rebrote, resultó un incremento cercano al $14 \%$, en la producción de forraje en base seca (Cuadro 2).

Cuadro 2. Producción de forraje en base seca según edad de rebrote. Estación Experimental Enrique Jiménez Núñez . Costa Rica. 1999.

\begin{tabular}{ccccc}
\hline Variable & $\begin{array}{c}\text { Error } \\
\text { estándar } \\
\text { de la } \\
\text { media }\end{array}$ & \multicolumn{4}{c}{ EDAD DE REBROTE (días) } \\
$\mathrm{Kg} \mathrm{MS/ha}$ & 885,5 & $3741,4 \mathrm{~b}$ & $4314,0 \mathrm{ab}$ & $4839,8 \mathrm{a}$ \\
\hline
\end{tabular}

MS= producción de forraje en base seca. Medias con letras diferentes son diferentes entre sí. $(p<0,01)$.

La producción de forraje en base seca también fue afectada por la fertilización nitrogenada ( $p<0,01$ ) (Figura 5). El comportamiento de dicho efecto, siguió el modelo de los rendimientos decrecientes, tal y como lo indica la literatura (Tisdale et al. 1985). 
Con respecto a este tipo de evaluaciones; la primera aplicación de $105 \mathrm{~kg}$ de N/ha fue la más eficiente con un incremento de $11,3 \mathrm{~kg}$ de forraje por kilogramo de nitrógeno aplicado. Con las aplicaciones siguientes la curva de respuesta se estabilizó y prácticamente no hubo aumentos.

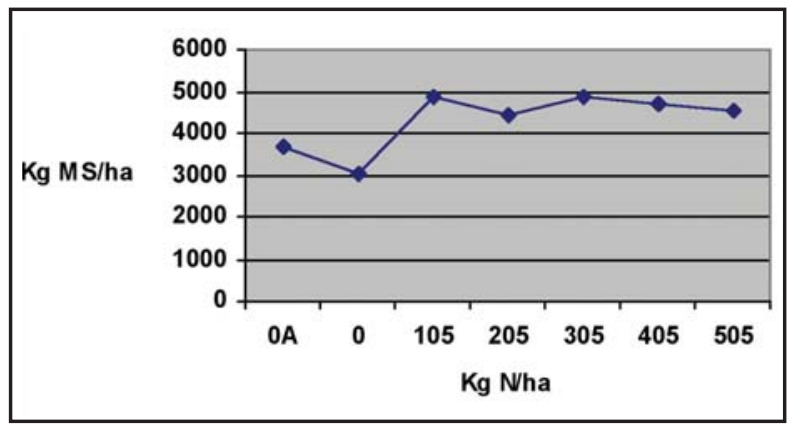

Figura 5 Efecto de la fertilización nitrogenada sobre la producción de forraje. Estación Experimental Enrique Jiménez Núñez. Costa Rica. 1999.

De nuevo en condiciones de baja disponibilidad de $\mathrm{N}$, en el testigo cero, se produce un efecto detrimental en la producción de forraje por la aplicación de fórmula completa (10-30-10).

\section{Contenido de fibra de la biomasa}

Los contenidos de fibra neutro detergente y fibra ácido detergente de los forrajes, son importantes porque están relacionados con la capacidad de consumo (Morales et al. 1989) y con la digestibilidad (Van Soest 1983) respectivamente.

En el presente estudio, el componente de fibra neutro detergente, no fue afectado por la edad de rebrote, ni por la fertilización del forraje (Cuadros 3 y 4). Sin embargo, la fracción de fibra ácida detergente presentó una tendencia a disminuir con el aumento en la edad de rebrote y muy ligeramente con la fertilización (Morales 1989).

Cuadro 3. Efecto de la edad de rebrote sobre el contenido de fibra en el forraje. Estación Experimental Enrique Jiménez Núñez. Costa Rica. 1999.

\begin{tabular}{|c|c|c|c|c|}
\hline \multirow[t]{2}{*}{ Variable } & \multirow{2}{*}{$\begin{array}{l}\text { Error } \\
\text { estándar } \\
\text { de la } \\
\text { media }\end{array}$} & \multicolumn{3}{|c|}{ EDAD DE REBROTE (días) } \\
\hline & & 40 & 50 & 60 \\
\hline$\%$ FND & 2,07 & 65,15 & 64,34 & 63,95 \\
\hline \multicolumn{5}{|l|}{ n.s. } \\
\hline$\%$ FAD & 1,25 & $46,48 a$ & $43,40 \mathrm{~b}$ & $44,05 \mathrm{~b}$ \\
\hline \multicolumn{5}{|c|}{$\begin{array}{l}\% \mathrm{FND}=\text { contenido de fibra neutro detergente; \% FAD= } \\
\text { contenido de fibra ácido detergente. } \\
\text { Medias con letras diferentes son diferentes entre sí ( } p< \\
0.01 \text {. }\end{array}$} \\
\hline
\end{tabular}

Cuadro 4. Efecto de la fertilización Nitrogenada sobre el contenido de fibra en el forraje. Estación Experimental Enrique Jiménez Núñez. Costa Rica. 1999.

\begin{tabular}{cccccccc}
\hline \multicolumn{7}{c}{ NIVELES DE FERTILIZACIÓN NITROGENADA (kg/ha) } \\
Variables & OA & $\mathbf{0}$ & $\mathbf{1 0 5}$ & $\mathbf{2 0 5}$ & $\mathbf{3 0 5}$ & $\mathbf{4 0 5}$ & $\mathbf{5 0 5}$ \\
$\%$ FND n.s. & 64,79 & 65,29 & 64,23 & 64,48 & 64,64 & 64,16 & 63,77 \\
$\%$ FAD & $45,83 \mathrm{ab}$ & $44,63 \mathrm{a}$ & $44,61 \mathrm{ab}$ & $44,09 \mathrm{~b}$ & $44,90 \mathrm{ab}$ & $44,42 \mathrm{~b}$ & $44,01 \mathrm{~b}$ \\
\hline
\end{tabular}

$\mathrm{OA}=$ testigo absoluto; \% FND= contenido de fibra neutro detergente; \% FAD= contenido de fibra ácido detergente. Medias con letras diferentes son diferentes entre si $(p<0,10)$. 


\section{CONCLUSIONES}

El proyecto marco de esta investigación y que se ha denominado "Industrialización del Heno en Costa Rica", busca establecer la tecnología para producir heno de buena calidad, en sistemas de producción bajo riego. El presente trabajo, aunque se realizó durante el período de lluvias, es válido, porque lo que pretendió buscar entre dos factores de manejo importantes, como son edad de rebrote del pasto y niveles de fertilización, los valores más cercanos al óptimo, para producir suficiente forraje de una calidad superior a la que se obtiene actualmente. Conociendo la respuesta del pasto a estos dos factores se podría avanzar entonces con mayor certeza y rapidez, en las evaluaciones propiamente de verano bajo riego.

Los resultados indican que, el pasto transvala puede ser manejado perfectamente a edades de rebrote de 40 a 60 días, con tasas de fertilización nitrogenada de alrededor de los $100 \mathrm{~kg}$ de nitrógeno por hectárea, por corte, para obtener producciones de forraje suficientes y de buena calidad, que permitan desarrollar la industria del heno de calidad en Costa Rica.

En sistemas de producción convencionales de heno de secano, es usual obtener unas 500 pacas de $17 \mathrm{~kg}$, a edades de rebrote que oscilan entre los tres a los siete meses. Se pueden considerar éstas como producciones altas, sin embargo, la calidad de este forraje es muy baja. Muchas veces, por ejemplo, el contenido de proteína cruda no alcanza el 4 $\%$, niveles que sí son usuales en la paja de arroz. Cuando los productores de heno utilizan algo de fertilizante aumentan la producción y un poco la calidad del forraje.

Durante el verano se disponen de 150 a 180 días secos, indispensables junto con las facilidades de riego existentes en el Distrito de Riego Arenal Tempisque, para desarrollar la industria del heno de calidad que se persigue. Esto quiere decir que la opción es la edad de rebrote máxima de 50 días y un máximo de
100 kg de nitrógeno por hectárea por corte para poder obtener con certeza tres cortes de heno en el verano, de un forraje de entre 8 y 9 $\%$ de proteína cruda. Los resultados de producción de forraje del presente estudio indican, que es factible alcanzar a producir alrededor de 300 pacas, de $17 \mathrm{~kg}$ promedio cada una, por hectárea, por corte.

Lo anterior significa que se pueden obtener 900 pacas de al menos $8 \%$ de proteína de pasto transvala por hectárea, en tres cortes en el verano. Ésto, más las 500 pacas de mala calidad, que igualmente este sistema obtendría del acumulado de forraje de invierno, como en los sistemas convencionales de secano, darían 1.400 pacas por hectárea (65\% de buena calidad) por año.

El presente es el primero de una serie de estudios que demuestran claramente, la posibilidad de desarrollar un sistema de producción de heno de buena calidad bajo riego, a través del cual, se triplica la producción y se duplica la calidad, en comparación con el sistema convencional de secano.

\section{LITERATURA CITADA}

Arroyo, L.; E. Araya ,M.; Ugalde, M. 2005. Análisis general del uso actual y potencial de la Estación Experimental Enrique Jiménez Núñez. Informe $N^{\circ}$ 1. INTA. San José, Costa Rica.52p.

Boyd, F.T.; Sank, S.C.; Smith, R.L.; Hodges, E.M.; West, S.H.; Kretschmer, A.K. ; Brolmann,J.B. and Moore,J.E. 1973. Transval Digitgrass a tropical forage resistant to: 1 . Swing nematodo, 2. Pangola Stunt Virus. Circular S22. Florida Agricultural Experiment Station. IFAS. University of Florida, Gainesville. 16 p.

EPA (U.S. Environmental Protection Agency). 2003. Ruminant Livestock and the Global Environment. http:/www.epa.gov/rlep/sustain.htm. 3 p.

Hancock, J.R. and Hargreaves, H.G. 1977. Precipitación, clima y potencial para producción agrícola en Costa Rica. International Irrigation Center. Agricultural and 
Irrigation Engineering Dept. Utah State University.160 p.

Leng, R.A. 1991. Application of biotechnology to nutrition of animals in developing countries. APHP 90. FAO. Roma. 14 p.

Leng, R.A. 1993. Quantitative Ruminant Nutrition - A Green Science. Aust. J. Agri. Research 44:363 - 380.

Milford, R. y Minson, D.J. 1966. Determinants of feeding value of pasture and Supplementary feed. Proceedings of the Australian Society of Animal Production. Vol. 6. p:319-329.

Morales, J.L. 1989. Managing the plant-animal interface in tropical legumegrass pastures. Ph.D. Dissertation. University of Florida. Gainesville, FL. 110 p.

Morales, J.L., van Horn, H.H. and Moore, J.E. 1989. Dietary interaction of cane molasses with source of roughage: intake and lactation effects. J. Dairy Sci. 72:2331-2338.

Morales, J.; Acuña, V. y Cruz, A. 2003. Industrialización del Heno de Calidad en Sistemas Bajo Riego en Costa Rica. INTA. Costa Rica. 60 p.

Nature Resource Council. 1984. Nutriente Requirements of Beef Cattle. National Academy Press. Washintong, USA.80 p.

Tisdale, S.L.; Nelson ,W.L. y Beaton, J.D. 1985. Soil fertility and fertilizers. 4 ed. Nueva York, USA. Macmillan.560 p.

Van Soest, P.J. 1967. Development of a comprehensive system of feed analyses and its application to forages. J. Anim. Sci. 26:119.

Van Soest, P.J. 1983 Nutritional ecology of the ruminant. O \& B Books. USA. p. 23-57. 